\title{
The Meta-Cybernetic Model of the Generic Human Mind
}

\author{
Benedykt Michal Josef Campbell Bieżanek* \\ Distinguished Researcher, Shropshire hill country England, UK
}

*Corresponding author: Benedykt Michal Josef Campbell Bieżanek, Distinguished Researcher, Shropshire hill country

England, UK

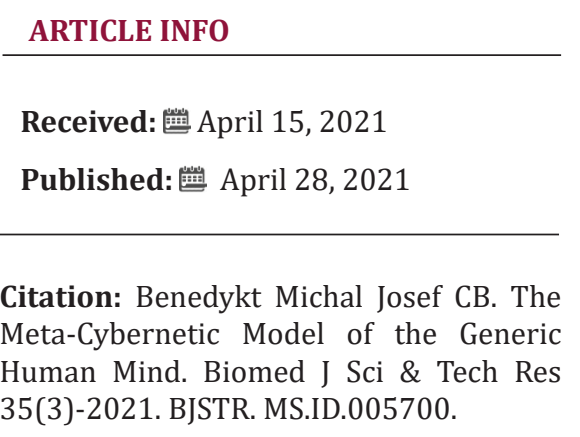

\section{ABSTRACT}

Abbreviations: FSE: Fantasy-Support-Environment; SPE: Sanity-Protection-Environment; EMB: Emotional Master Being

\section{Opinion}

A number of problems have been encountered with healthy humans who appear to be under no undue stress, perfectly welleducated, well-trained and in perfect mental health and even working together as a part of a small team and yet still making catastrophic errors resulting in them making serious mistakes. The Meta-Cybernetic Model describes the human mind as consisting of world-views cocooned within a Fantasy-Support-Environment (FSE). The FSE employs about seven orders of magnitude more neural mass than that relatively tiny neural mass that might hold the core fantasy world-view or fantasy view of theoretical physics or whatever else it is that the mind is interested in trying to think about. We can say; "he was only human; he just made a mistake". In meta-cybernetics we say "not good enough, kindly explain why it is human to make mistakes".

The Meta-Cybernetic Model is useful because it has empowered a bridge between meta-physics and theoretical physics that has resulted in The Gauss-Newton Quantum-Relativity which is merely an enhanced flavor of Einstein's special theory of relativity. It has also been used to build a bridge between the superior higher arithmetical language of Gauss and the faulty higher arithmetical language we have all been taught by others. An alternative name for the fantasy-support-environment is the Sanity-Protection-

Environment (SPE), the practitioner may challenge his fantasies but only in the hope of finding a more satisfying fantasy. The practitioner must realize that with some unknown critical fantasy destroyed, he would become stark raving insane and move into a very bad place indeed. We share a name for that very bad place; we call that the Schizophrenic altered mind-state.

\section{A Practical Case Study for Psychiatric Practitioners}

It is generally believed by psychiatrists that psychoanalysis and counseling is unhelpful in the treatment of Schizophrenia. There is no effective treatment for Schizophrenia and the psychiatric practitioner will typically administer pharmaceuticals which have the effect of making the sufferer less of a danger and less of a difficulty for others while at the same time tending to destroy the basic humanity that the sufferer can retain. The treatment is a doctrine of despair and the prognosis is for the sufferer to die with the mental impairment. Meta-Cybernetics can help here, even though the meta-cybernetician has no knowledge of psychiatric practice and has not studied any medical science. There is the case study of John Forbes Nash. Nash was a brilliant mathematician who contracted a serious case of Schizophrenia in his mid-twenties and was gone away into the Schizophrenic altered-mind-state for about thirty years. 
Nash recovered completely and even explained the actions that the Schizophrenic-sufferer can take to get back to normal in a few simple sentences. The obvious implied treatment therapy is missed by the psychiatric profession because they would prefer not to listen to the subject Nash. They prefer to regard the Nash recovery case as an exception and an isolated miracle rather than practical treatment advice that they could all pay attention to. Nash explains how he chose to recover and he fully explained his own treatment of himself. He decided to very painfully withdraw from all medication and stop paying any attention at all to all of the strange voices that existed within his mind. Over a gradual period of the last three years of his thirty-year mental excursion, Nash's friends were amazed. After three years in recovery, Nash was fully back from the brink as good as he ever was with the exception that his friends reported that he was a much nicer person to interact with in his recovered mind-state than he was before he became ill.

He was so nice to be with in the recovered mind-state that his divorced wife stopped merely caring for him and thirty years after divorcing him, she then happily remarried him. The obvious treatment protocol indicated for the psychiatric practitioner is to gently explain the Nash case study to the sufferer. Remaining in the Schizophrenic altered-mind-state is a choice and not a permanent condition. The only person who can do anything about it is the sufferer himself and only if he chooses to see the reason why he should change back to how he was before and then merely follow the genius pioneer Nash slowly back into our "real world".

\section{The Neurological Mind-Model}

The neurological model is very simple and comes from an examination of artificial intelligence. The neurological mass is highly complex involving a large number of interconnected complex intelligent adaptive systems. The conscious Emotional Master Being (EMB) receives neurological information from the primary senses only after a vast array of complex subconscious neural network processing, filtering, inferencing and path blocking. The EMB is looking for logically satisfying or emotionally satisfying confirmation in the source data. The networks become trained to please the EMB and to avoid giving emotional or logical pain to the Emotional Master Being. The networks are utterly enslaved to the logical and emotional needs of the emotional and logical conscious master. To give one a feel for the level of complexity here, 35-years ago I helped to build an artificial anesthetist.

This was a very successful demonstration project. Our AI anesthetist was trained by an expert system containing every fact that human anesthetists asserted to be true, garnered from a sample of 1,500 practicing anesthetists. We paid no attention at all to obvious contradictions in the human belief set or to the highly varied work quality results of the human practitioners within the sample. The AI anesthetist learned the trade over a synthetic career of about 1.5 million human years of experience, with highly variable synthetic models of patients, surgical procedures, operating surgeons, and theater recovery nursing personnel. The simulations ran at about 300,000 times real-time and were left to continue 24/7 for nearly one year of elapsed apparent training time. Once we were confident that our AI anesthetist was good to go, a volunteer team of real surgeons, a real supervising anesthetist and a large number of volunteer patients tried "him" out for real. Our AI anesthetist was embarrassingly good, clearly outperforming the very best of the humankind.

The entire project was only a demonstration, and the AI anesthetist was then quietly decommissioned. If we thought that we are important to human survival on account of our great human ability at highly complex tasks, then we need to completely forget that silly thought all together. My point in telling us of this account is only to point out that a typical human mind contains a network with about 100 billion neurons and our AI anesthetist worked with just seven artificial neurons. The human mind is not just more complex than any such silly little machine, he is catastrophically more complex than all of our wildest dreams.

\section{The Electrical Engineer's Conclusion}

So, my question to my fellow electrical engineers is not so much; "how can we explain our great human intelligence?" But rather; "how can we explain our unbelievably poor actual performance?" The answer turns out to be very simple indeed. For every neuron that is actually engaged in conscious human logical deduction and world-view model building, there might be ten million involved with the provision of the Fantasy-Support-Environment (FSE). In the Meta-Cybernetic Model, we describe the Emotional Master Being as existing deeply cocooned within his own private FantasySupport-Environment. There is not that much that is that private about our personal FSE, because we have spent the first twenty odd years of our life acquiring and learning roughly the same FSE that our teachers and parents had. That FSE was built by a long chain of FSE stretching all the way back to perhaps the first ape who accidentally found out that he could break and form softer stones with hard stone in his hand over a hard stone anvil.

We might call that the evolution of human thought, but there is nothing gradual about it, the changes occur in tiny fantastic accidental jumps. One moment apes could not break stones at all, and a few months later, even the very young apes in the genius ape-tribe were doing little else. How did the author uncover the Meta-Cybernetic Model? The same way that our ape did, one day he accidentally just put two and two together, and then bingo, four there it was. 


\section{ISSN: 2574-1241}

DOI: 10.26717/BJSTR.2021.35.005700

Benedykt Michal Josef CB. Biomed J Sci \& Tech Res

(c) $(2)$ This work is licensed under Creative

Submission Link: https://biomedres.us/submit-manuscript.php

$\begin{array}{ll}\text { BIOMEDICAL } & \text { Assets of Publishing with us } \\ \text { RESEARCHES } & \text { Global archiving of articles } \\ & \text { - Immediate, unrestricted online access } \\ & \text { - Rigorous Peer Review Process } \\ & \text { - Anthors Retain Copyrights }\end{array}$

